\title{
Insulin Sensitivity, Depression/Anxiety, and Physical Fitness in At-Risk Adolescents
}

\section{(c) (1) (우 $\odot$}

\author{
Authors \\ Stephanie M. Bruggink'1, Lauren Berger Shomaker ${ }^{1}$, Nichole R. Kelly², 3, 4, Bart E. Drinkard5, Kong Y. Chen ${ }^{6}$, \\ Robert J. Brychta ${ }^{6}$, Omni Cassidy3, ${ }^{3}$, Andrew P. Demidowich ${ }^{3}$, Sheila M. Brady3, Marian Tanofsky-Kraff3, 4 , Jack A. Yanovski, 3
}

\section{Affiliations \\ 1 Human Development \& Family Studies, Colorado State University College of Health and Human Sciences, Fort Collins, Colorado, United States \\ 2 Counseling Psychology and Human Services and the Prevention Science Institute, College of Education, University of Oregon, Eugene, Oregon, United States \\ 3 Section on Growth and Obesity, Division of Intramural Research, National Institute of Child Health and Human Development, Bethesda, Maryland, United States \\ 4 Uniformed Services University of the Health Sciences, Medical and Clinical Psychology, Bethesda, Maryland, United States \\ 5 Rehabilitation Medicine Department, Mark O Hatfield Clinical Research Center, Bethesda, Maryland, United States \\ 6 Diabetes, Endocrinology, and Obesity Branch, National Institute of Diabetes, Digestive and Kidney Diseases, Bethesda, Maryland, United States}

\section{Key words}

adolescents, physical fitness, depression, anxiety, insulin sensitivity

$\begin{array}{ll}\text { received } & 17.10 .2018 \\ \text { revised } & 14.02 .2019 \\ \text { accepted } & 25.03 .2019\end{array}$

\section{Bibliography}

DOI https://doi.org/10.1055/a-0889-8653

Published online: 2019

Sports Medicine International Open 2019; 3: E40-E47

(c) Georg Thieme Verlag KG Stuttgart · New York

ISSN 2367-1890

\section{Correspondence}

Dr. Lauren Berger Shomaker, PhD

Human Development \& Family Studies,

Colorado State University, College of Health and

Human Sciences,

1570 Campus Delivery,

80523 Fort Collins, CO

United States

Tel.: + 1/970/4913-217, Fax: + 1/970/4917-975

lauren.shomaker@colostate.edu

\begin{abstract}
Poor physical fitness contributes to the early progression of cardiometabolic disease, yet the physiological and psychological factors underpinning poor fitness in at-risk adolescents are not well understood. In this study, we sought to determine the relationship of physical fitness with two developmental phenomena of adolescence, insulin resistance and depression/ anxiety symptoms among at-risk youth. We conducted secondary data analyses of 241 overweight or obese adolescents (12-17 years), drawn from two study cohorts. Insulin sensitivity index was derived from oral glucose tolerance tests. Adolescents self-reported depressive symptoms and anxiety symptoms on validated surveys. A walk/run test was administered to determine perceived exertion and physical fitness (distance traveled). Insulin sensitivity was positively associated with walk/ run distance $(b=0.16, P<0.01)$, even after accounting for all covariates. Anxiety symptoms were inversely related to perceived exertion ( $b=-0.11, P<0.05)$, adjusting for covariates. These findings suggest that insulin resistance and anxiety symptoms are associated with different dimensions of physical fitness in overweight or obese adolescents and could both potentially contribute to declining fitness and worsening metabolic outcomes in at-risk youth.
\end{abstract}

The progressively earlier onset of preventable lifestyle-related diseases has become an alarming public health issue among adolescents in the United States. In particular, type 2 diabetes is increasingly prevalent in adolescents, especially adolescent females from historically disadvantaged racial/ethnic groups [4]. Early onset of type 2 diabetes is associated with a more severe and aggressive disease course, necessitating a better understanding of the potentially modifiable precursors to cardiometabolic disease [23]. 
A modifiable risk factor, poor physical fitness, caused by insufficient moderate-to-vigorous physical activity and high amounts of sedentary behavior [8], is correlated with reduced insulin sensitivity in adolescents, above and beyond the effects of adiposity and body mass index (BMl; $\mathrm{kg} / \mathrm{m}^{2}$ ) [1]. Adolescence is marked by a notable decrease in moderate-to-vigorous physical activity and an increase in sedentary behavior, especially in girls from historically disadvantaged racial/ethnic groups [15,24], potentially exacerbating the pubertal increases in insulin resistance [14]. Conversely, the decreases in insulin sensitivity accompanying puberty also have been posited to lead to fatigue, triggering adolescents' withdrawal from physical activity and subsequently, poorer fitness [3].

In addition, adolescence is notable for a rise in affective disturbances, including internalizing symptoms of depression and anxiety [18], which may contribute to declining physical activity and fitness during this stage of development [24]. In adults, symptoms of depression and anxiety have been associated with obesity, physical inactivity, poor physical fitness, and type 2 diabetes [19, 28, 34]. Although there is a smaller body of literature examining these associations among adolescents, existing data suggest that adolescent depressive symptoms relate to less reported physical activity over time, possibly due to the vegetative symptoms of depression such as fatigue and psychomotor retardation [13]. Social isolation, which frequently accompanies depression and anxiety symptoms, also may play a role [9].

In the current study, we sought to evaluate how both insulin sensitivity and symptoms of depression and anxiety were associated cross-sectionally with objectively-measured physical fitness during walk/run testing in adolescents at heightened risk for cardiometabolic disease. We hypothesized that insulin sensitivity would be positively associated with physical fitness, whereas depression and anxiety symptoms would be inversely associated with fitness. We anticipated that these associations would exist independently of current adiposity. We examined these aims and hypotheses in two adolescent cohorts; one was a group of overweight/obese females with elevated depression symptoms and a family history of type 2 diabetes, and the other was a group of males and females with obesity and obesity-related health comorbidities. Despite some expected descriptive differences between the samples by study design (e. g., depression level and degree of obesity/BMI standard score), we anticipated that the relations of insulin sensitivity and internalizing symptoms with physical fitness would be similar across cohorts.

\section{Materials and Methods}

\section{Study participants}

This study represents a secondary data analysis of two convenience samples used to address the current research questions.

\section{Cohort 1}

Cohort 1 participants were recruited to take part in a behavioral trial for the prevention of type 2 diabetes (NCT 01425905); the main outcomes have been reported [29,30]. Participants were adolescent girls ages $12-17$ years who were in good general health, other than being overweight or obese (BMI $\geq 85^{\text {th }}$ percentile for age and sex) and all reported a family history of diabetes. Adolescents reported mild-to-moderate depressive symptoms, determined as a total score $\geq 16$ on the Centers for Epidemiologic Studies Depression Scale [26]. Volunteers were excluded if they had current psychiatric symptoms requiring treatment; fasting glucose $>126 \mathrm{mg} / \mathrm{dL}$; 2 -hour glucose $>200 \mathrm{mg} / \mathrm{dL}$; pregnancy; use of medication affecting insulin sensitivity, weight, or mood; and/or were involved in current psychotherapy or weight loss treatment. The present analysis uses data only from the baseline assessment.

\section{Cohort 2}

Cohort 2 participants were recruited for a medication trial for the treatment of obesity (NCT00001723). Other exercise-related outcomes have been reported elsewhere [6, 25, 27, 31]. Participants were non-Hispanic Black and White adolescent boys and girls 12-17 years with obesity (BMI $\geq 95^{\text {th }}$ percentile for age and sex) and $\geq 1$ obesity-related health co-morbidity, including hypertension, hyperlipidemia, sleep apnea, hepatic steatosis, insulin resistance, impaired glucose tolerance, and/or type 2 diabetes. Volunteers were excluded for current or past medical problems that would impair exercise testing performance, medication use for weight loss, psychiatric symptoms that necessitated a referral and would impede compliance, and pregnancy or nursing in females. In the present analysis, we included data only from the baseline assessment.

\section{Procedures and measures}

Unless otherwise noted, the same method was used in both samples. All assessments took place in the Mark O. Hatfield Clinical Research Center of the National Institutes of Health. Procedures were approved by the Institutional Review Board of the Eunice Kennedy Shriver National Institute of Child Health and Human Development and were consistent with the ethical standards of Sports Medicine International Open [11]. Parents/guardians provided written informed consent, and adolescents provided assent to participate.

\section{Anthropometric measurements}

Height was measured in triplicate by stadiometer and averaged. Fasting weight was assessed using a calibrated digital scale. Height and weight were used to compute BMI $\left(\mathrm{kg} / \mathrm{m}^{2}\right)$, BMI standard score $(\mathrm{z})$, and $\mathrm{BMI}$ percentile for age and sex. Fat and lean mass $(\mathrm{kg})$ were measured using dual-energy $x$-ray absorptiometry using either a Hologic QDR-4500A instrument (Hologic Inc., Waltham, MA, USA) or iDXA (GE Healthcare, Madison, WI, USA).

\section{Puberty}

Pubertal development was determined from physical examination by an endocrinologist or nurse practitioner using Tanner staging derived from breast development in females and testicular volume in males [20,21].

\section{Physical fitness and perceived exertion}

Physical fitness was measured using an individual 12-minute walk/ run shuttle test, running back and forth in a hallway, that has been shown to correlate with cardiorespiratory fitness as measured dur- 
ing a maximal cycle ergometry test in adolescents with obesity [6]. Participants were instructed to give their best effort by covering as much distance as possible in $12 \mathrm{~min}$. Total distance traveled was measured with a measurement wheel. Perceived exertion, referring to adolescents' perceptions of degree of effort and intensity level of their physical activity, was assessed upon completion of the fitness test with the 20-point Borg Rating of Perceived Exertion Scale (range $=6-20$ ) [2]. Participants were shown a diagram to familiarize themselves with the rating of perceived exertion scale.

\section{Depression and anxiety symptoms}

Adolescents completed widely-used, validated survey instruments to assess depression and anxiety symptoms in this age group. Adolescents completed questionnaires in a private room and were instructed that surveys would ask about their feelings and behavior. Depressive symptoms were determined using adolescents' reports on the reliable and well-validated Children's Depression Inventory, a 27-item survey with the total score calculated as the sum of all items (range $=0-54$ ) [16]. Anxiety symptoms were measured using youth's report on the 20-item State-Trait Anxiety Inventory for Children (STAI-C)-Trait Version, a widely used reliable and valid measure, calculated as the sum of all items (range $=20-60$ ) [32].

\section{Insulin sensitivity}

Testing to assess insulin sensitivity was performed in a pediatric research clinic in the morning following an overnight fast, in which adolescents were asked to refrain from food or drink other than water, after 10:00 pm the evening before the visit. A 2-hour oral glucose tolerance test (OGTT) was used to determine insulin sensitivity, calculated as Insulin Sensitivity Index (ISI) using reduced time points of insulin and glucose at 0 and 120 min [5]. Using only fasting samples, we also determined insulin sensitivity with quantitative insulin sensitivity check index (QUICKI) [10] and insulin resistance with the homeostasis model assessment of insulin resistance (HOMA-IR) formulae [22].

\section{Statistical analyses}

Analyses were conducted with SPSS version 23. Data were cleaned by winsorizing outliers to fall 1.5 times the interquartile range below the $25^{\text {th }}$ percentile or above the $75^{\text {th }}$ percentile. Characteristics of cohorts 1 and 2 were compared using chi-square for categorical variables and independent samples t-tests for continuous variables. Multiple hierarchical regression models using listwise deletion to handle missing data $(0.6 \%)$ were used to examine the associations of insulin sensitivity, depressive symptoms, and anxiety symptoms (independent variables) with physical fitness, including perceived exertion and total distance traveled (dependent variables). We included the following covariates in step 1 of the models: race/ethnicity, age, puberty, height, lean mass, percent adiposity, sex, and study cohort. In step 2, ISI, QUICKI, or HOMA-IR were entered in separate models. In step 3, depression and anxiety symptoms were added. We combined cohorts 1 and 2 in the analyses; however, given demographic differences in the cohorts, by design, we also analyzed the models separately, stratified by study cohort. Significance was determined as a $\mathrm{P}<0.05$. Effect sizes were estimated with Cohen's $\mathrm{d}(\sim 0.20$ : small, $\sim 0.50$ : medium, $\geq 0.80$ : large).

\section{Results}

\section{Sample}

Characteristics of the total sample $(\mathrm{N}=241)$, as well as differences between cohort $1(n=100)$ and cohort $2(n=141)$, are displayed in - Table 1. Average (SD) age of participants was 14.67 (1.46) years, with no age difference between samples $(P=0.15)$.

\section{Perceived exertion}

- Table 2 displays a summary of regression analyses for perceived exertion during walk/run fitness testing. Within the combined cohort, anxiety symptoms were negatively associated with perceived exertion ( $P=0.02$; Cohen's $d=0.10)$, after accounting for all covariates. When the analyses were stratified by cohort, anxiety symptoms remained significant in the model including cohort $1(\mathrm{P}<0.05$; Cohen's $d=0.23$ ).

\section{Walk/run distance}

- Table 3 summarizes the results for the regression models for walk/run distance traveled. Accounting for all covariates, insulin sensitivity by ISI was significantly, positively associated with walk/ run distance $(P<0.01$; Cohen's $d=0.16)$. ISI explained $2 \%$ of the unique variance in walk/run distance $(P<0.01)$, after accounting for covariates. Parallel effects were observed for insulin sensitivity measured via QUICKI (Cohen's $d=0.23$ ) and insulin resistance estimated with HOMA-IR (Cohen's $d=0.25$ ). When we also explored controlling for perceived exertion in these models predicting walk/ run distance, the effects of insulin sensitivity/resistance remained significant.

When analyses were stratified by study cohort, results were similar. In cohort 1, the associations of QUICKI (Cohen's d = 0.25 ) and HOMA-IR (Cohen's $d=0.31$ ) remained significantly associated with walk/run distance ( $P \leq 0.01$ ), but the association of ISI with walk/ run distance was attenuated $(P=0.13$; Cohen's $d=0.14$ ). In cohort 2 , the effects for all three indices of insulin sensitivity/resistance remained significant predictors of walk/run distance $(P<0.01$; Cohen's $d=0.17-0.23$ ).

In addition, after including covariates and insulin sensitivity in the model, depressive symptoms were significantly, inversely associated with walk/run distance in cohort 2 only $(P<0.05$; Cohen's $d=0.19)$.

\section{Discussion}

In the current paper, we conducted a secondary cross-sectional data analysis of two adolescent cohorts at heightened risk for cardiometabolic disease. The aim was to determine the relationship of two developmental phenomena of adolescence - reduced insulin sensitivity and increased depression and anxiety symptoms - to physical fitness, measured during walk/run testing. In the total combined sample, anxiety symptoms related to less perceived exertion, and insulin resistance related to less distance traveled, with some slight, but potentially meaningful, differences by cohort.

An inverse association of anxiety symptoms with adolescents' perceived effort during physical activity was apparent in cohort 1 , the female-only cohort. These participants had more elevated symptoms of depression and anxiety compared to cohort 2 . Our results are con- 
- Table 1 Characteristics of the total sample and differences in characteristics between cohorts 1 and 2 .

\begin{tabular}{|c|c|c|c|c|c|c|c|}
\hline \multirow[b]{3}{*}{ Characteristic } & \multicolumn{2}{|c|}{ Total Sample } & \multicolumn{2}{|c|}{ Cohort 1} & \multicolumn{2}{|c|}{ Cohort 2} & \multirow[b]{3}{*}{$\mathbf{P}$} \\
\hline & \multicolumn{2}{|c|}{$N=241$} & \multicolumn{2}{|c|}{$n=100$} & \multicolumn{2}{|c|}{$n=141$} & \\
\hline & $\mathrm{M} \pm \mathrm{SD}$ & $n, \%$ & $\mathrm{M} \pm \mathrm{SD}$ & $\mathrm{n}, \%$ & $\mathrm{M} \pm \mathrm{SD}$ & $\mathrm{n}, \%$ & \\
\hline Sex & & & & & & & $<0.001$ \\
\hline Male & & $48,19.9 \%$ & & $0,0 \%$ & & $48,34.0 \%$ & \\
\hline Female & & $193,80.1 \%$ & & $100,100 \%$ & & $93,66.0 \%$ & \\
\hline Race/ethnicity & & & & & & & $<0.001$ \\
\hline Non-Hispanic Black & & $142,58.9 \%$ & & $59,59.0 \%$ & & $83,58.9 \%$ & \\
\hline Non-Hispanic White & & $76,31.5 \%$ & & $18,18.0 \%$ & & $58,41.1 \%$ & \\
\hline Hispanic & & $12,5.0 \%$ & & $12,12.0 \%$ & & $0,0 \%$ & \\
\hline Multiple or Other & & $11,4.6 \%$ & & $11,11.0 \%$ & & $0,0 \%$ & \\
\hline Age, $y$ & $14.67 \pm 1.46$ & & $14.83 \pm 1.50$ & & $14.56 \pm 1.42$ & & 0.15 \\
\hline BMI z score & $2.31 \pm 0.47$ & & $1.99 \pm 0.48$ & & $2.53 \pm 0.32$ & & $<0.001$ \\
\hline Body fat, $\%$ & $46.35 \pm 6.57$ & & $43.14 \pm 5.76$ & & $48.63 \pm 6.16$ & & $<0.001$ \\
\hline \multicolumn{8}{|l|}{ Internalizing symptoms } \\
\hline Depressive symptoms & $9.87 \pm 6.65$ & & $13.57 \pm 6.10$ & & $7.24 \pm 5.73$ & & $<0.001$ \\
\hline Anxiety symptoms & $35.03 \pm 7.54$ & & $38.80 \pm 6.69$ & & $32.35 \pm 6.96$ & & $<0.001$ \\
\hline \multicolumn{8}{|l|}{ Insulin sensitivity } \\
\hline ISI & $2.67 \pm 1.71$ & & $2.80 \pm 1.90$ & & $2.59 \pm 1.57$ & & 0.36 \\
\hline QUICKI & $0.30 \pm 0.02$ & & $0.31 \pm 0.03$ & & $6.05 \pm 3.33$ & & 0.36 \\
\hline HOMA-IR & $5.86 \pm 3.30$ & & $0.30 \pm 0.02$ & & $5.59 \pm 3.26$ & & 0.28 \\
\hline \multicolumn{8}{|l|}{ 12-Min. Walk-Run Test } \\
\hline Distance, $\mathrm{m}$ & $1176.64 \pm 208.20$ & & $1220.29 \pm 216.40$ & & $1146.68 \pm 197.25$ & & 0.01 \\
\hline Perceived exertion & $11.05 \pm 3.50$ & & $14.42 \pm 2.02$ & & $8.31 \pm 1.47$ & & $<0.001$ \\
\hline
\end{tabular}

sistent with previous research suggesting that women with obesity experience lower energy and report less pleasure from exercise than women without obesity [7]. The current findings suggest that anxiety may be a barrier to engagement in physical activity in heavier adolescents, particularly in girls. Adolescence is notable for increases in anxiety and body dissatisfaction, and adolescent girls who are heavier report greater anxiety and more anxiety specific to body image and physique than females who are leaner [33]. Exertion relates to likelihood to achieve fitness, and declining physical fitness is a key contributor in the progressive course of cardiometabolic disease onset [1]; thus, strategies for directly addressing this distinct psychological barrier (e. g., altering the contextual features and/or type of delivery of physical activity interventions) warrant consideration.

In contrast, depression symptoms related to less distance traveled in cohort 2 only, the sample of adolescent girls and boys with obesity and obesity-related health co-morbidities. This effect is consistent with previous studies showing a relationship of depression to cardiorespiratory fitness and to prospective declines in physical activity over time in adolescents [13, 31]. In theory, the vegetative symptoms of depression, including fatigue, low energy, sleep disruption, appetite dysregulation, and anhedonia or lack of pleasure in daily activities, are proposed to contribute to withdrawal from physical activities, which, in turn, further worsens depressed mood [13]. Indeed, behavioral activation - scheduling and doing activities even when one does not feel like it - is an evidence-based interven- tion for preventing and treating adolescent depression [12]. Addressing symptoms of depression may have the potential to encourage engagement in physical activity that leads to improvements in physical fitness, and in turn, lessens cardiometabolic risk independent of weight loss. In the current study, the lack of association of depressive symptoms with physical fitness in cohort 1 cannot be determined with certainty, but one likely explanation is that this null effect may be explained by the more restricted variability in depression symptoms in cohort 1 , all of whom were girls reporting some degree of elevated affective disturbance.

Insulin sensitivity, assessed across multiple indices (ISI, HOMAIR, QUICKI), was positively associated with walk/run distance traveled. These associations were observed consistently in the combined sample and across both cohorts examined separately, notably after controlling for body composition and other possible confounds (with the exception that the relationship of ISI to walk/run distance was slightly attenuated in cohort 1). The relationship of insulin sensitivity and physical fitness may be explained by several possible underlying mechanisms. One possibility is that poorer physical fitness may worsen insulin sensitivity in youth independent of adiposity [1]. Youth with recent-onset T2D, for instance, have less moderate-to-vigorous activity and spend more time engaging in sedentary behaviors than youth with obesity but no T2D, suggesting a possible putative role for physical activity and fitness in T2D onset, regardless of degree of adiposity [17]. Alternatively, it is pos- 


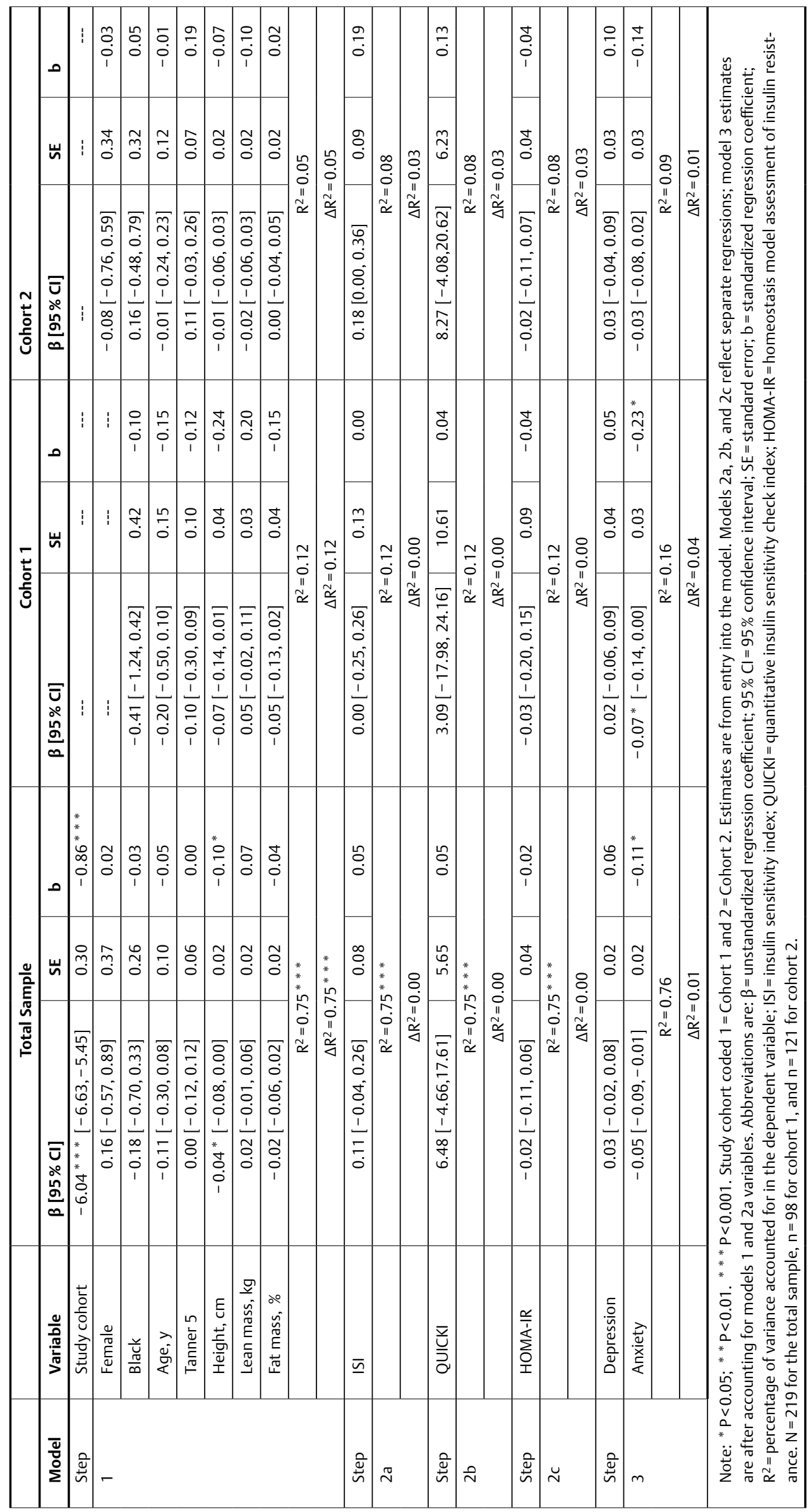




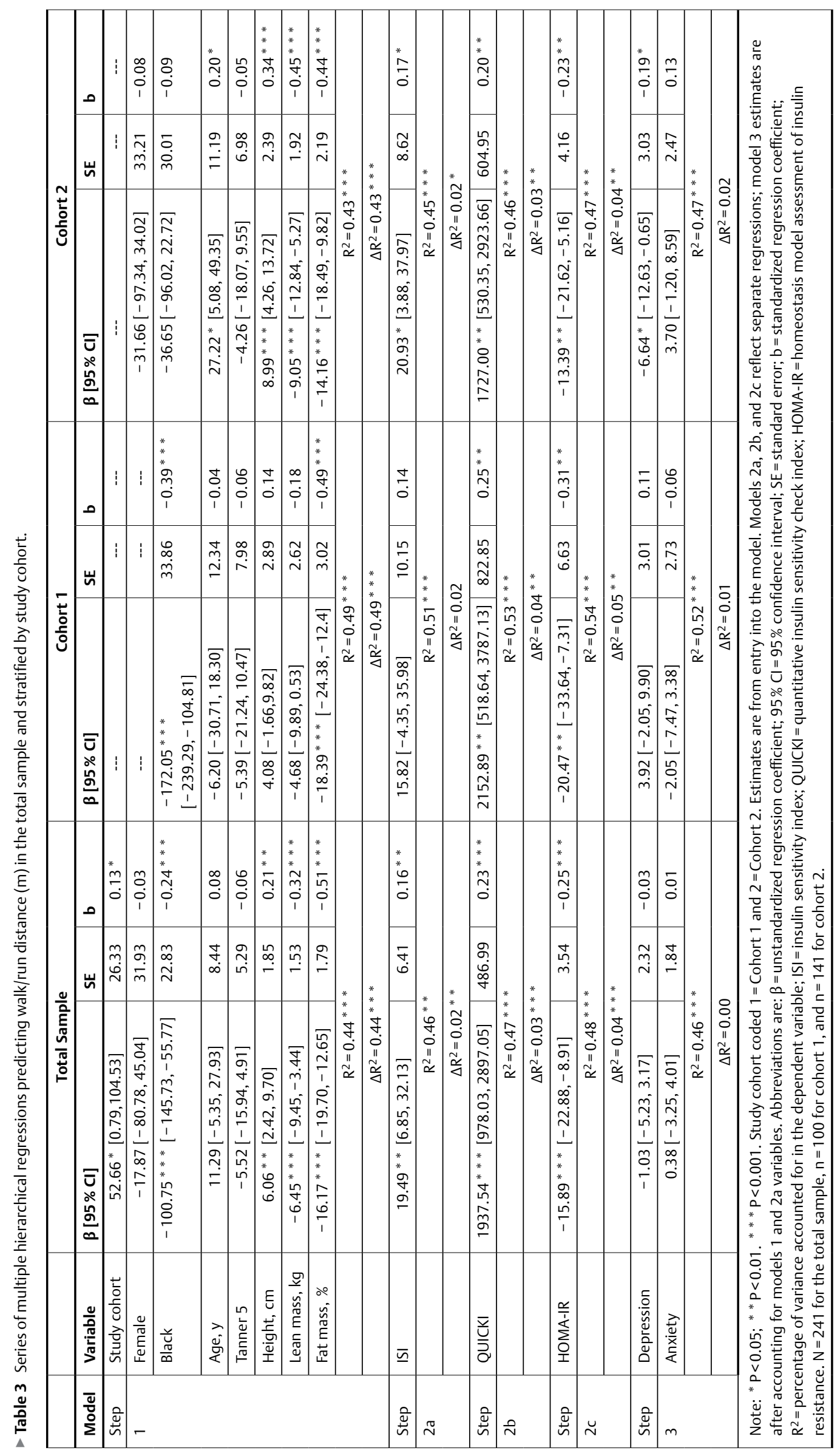


sible that poor insulin sensitivity may contribute to less physical activity due to physiological consequences such as fatigue [3]. If the insulin sensitivity-physical fitness connection represents a bidirectional relationship, then theoretically, intervening to improve insulin sensitivity (e.g., through pharmacological agents) might enhance adolescent's willingness to engage in physical activity, thereby improving their fitness, just as improving fitness would be anticipated to improve insulin resistance. These alternative hypotheses require experimental testing in future research.

Limitations to the present study include the cross-sectional and correlational design, making it impossible to determine the causal direction of effects. All of the effects we did observe for the relationships of psychological symptoms and insulin sensitivity with perceived exertion or walk/run distance were small, underscoring the multitude of factors that are associated with physical activity in overweight or obese adolescents. Generalizability of the observed effects are limited to adolescents at-risk for cardiometabolic disease and may or may not apply to adolescents with lower risk of disease. Non-Hispanic Black and non-Hispanic White adolescents were also disproportionally sampled and there was limited representation of Hispanic adolescents or other racial/ethnic groups at heightened risk for obesity and metabolic disorders. Similarly, the cohorts did not include younger adolescents at a less advanced pubertal stage. This feature made the participants largely homogenous for the late pubertal stage, and thus, we could not ascertain or isolate the distinctive association of pubertal development or timing with the variables of interest examined in this study. Though walk/run testing relates to cardiorespiratory fitness [6], an important risk factor for cardiometabolic disease, we did not evaluate habitual physical activity. Future work should consider ambulatory physical activity monitoring in relation to insulin sensitivity and depression/anxiety symptoms to determine whether the observed associations apply to physical activity.

The current study supports a role for both insulin sensitivity and affective disturbance in dimensions of physical fitness among adolescents at high-risk for cardiometabolic disease. Most adolescents, and particularly those who are overweight or obese, do not achieve the recommended $60 \mathrm{~min}$ per day of moderate-to-vigorous physical activity [35]. Considering developmentally-sensitive factors may be relevant in the design of more effective interventions to address a strong need for increased physical activity and physical fitness in at-risk adolescents.

\section{Financial Support}

K99HD069516 and R00HD069516 (LBS) from NICHD, NIH and Intramural Research Program Grant 1ZIAHD000641 (JAY) from NICHD with supplemental funding from the NIH Bench to Bedside Program (LBS, MT-K, JAY), Office of Behavioral and Social Sciences Research (JAY), and the NIH Office of Disease Prevention (JAY). Clinical trial reg. no: NCT01425905, NCT00001723, clinicaltrials.gov.

\section{Conflict of Interest}

None of the authors has a conflict of interest. J. A. Yanovski and B.E. Drinkard are Commissioned Officers in the U.S. Public Health Service. The opinions and assertions expressed herein are those of the authors and are not to be construed as reflecting the views of the Public Health Service, the Department of Health and Human Services, USUHS, or the U.S. Department of Defense.

\section{References}

[1] Berman LJ, Weigensberg M], Spruijt-Metz D. Physical activity is related to insulin sensitivity in children and adolescents, independent of adiposity: A review of the literature. Diabetes Metab Res Rev 2012; 28: 395-408

[2] Chen M], Fan X, Moe ST. Criterion-related validity of the Borg ratings of perceived exertion scale in healthy individuals: A meta-analysis. J Sports Sci 2002; 20: 873-899

[3] Coyle EF, Hagberg JM, Hurley BF, Martin WH, Ehsani AA, Holloszy JO. Carbohydrate feeding during prolonged strenuous exercise can delay fatigue. J Appl Physiol Respir Environ Exerc Physiol 1983; 55: 230-235

[4] Dabelea D, Mayer-Davis EJ, Saydah S, Imperatore G, Linder B, Divers ], Bell R, Badaru A, Talton JW, Crume T, Liese AD, Merchant AT, Lawrence JM, Reynolds K, Dolan L, Liu LL, Hamman RF. Search for diabetes in youth study. Prevalence of type 1 and type 2 diabetes among children and adolescents from 2001-2009. JAMA 2014; 311: 1778-1786

[5] DeFronzo RA, Matsuda M. Reduced time points to calculate the composite index. Diabetes Care 2010; 33: e93

[6] Drinkard B, McDuffie J, McCann S, Uwaifo Gl, Nicholson J, Yanovski JA. Relationships between walk/run performance and cardiorespiratory fitness in adolescents who are overweight. Phys Ther 2001; 81: 1889-1896

[7] Ekkekakis P, Lind E, Vazou S. Affective responses to increasing levels of exercise intensity in normal-weight, overweight, and obese middleaged women. Obesity (Silver Spring) 2010; 18: 79-85

[8] Ganley KJ, Paterno MV, Miles C, Stout J, Brawner L, Girolami G, Warren M. Health-related fitness in children and adolescents. Pediatr Phys Ther 2011; 23: 208-220

[9] Graham D], Wall MM, Larson N, Neumark-Sztainer D. Multicontextual correlates of adolescent leisure-time physical activity. Am J Prev Med 2014; 46: 605-616

[10] Gungor N, Saad R, Janosky J, Arslanian S. Validation of surrogate estimates of insulin sensitivity and insulin secretion in children and adolescents. J Pediatr 2004; 144: 47-55

[11] Harriss D], Macsween A, Atkinson G. Standards for ethics in sport and exercise science research: 2018 update. Int J Sports Med 2017; 38: 1126-1131

[12] Hetrick SE, Cox GR, Witt KG, Bir J], Merry SN. Cognitive behavioural therapy (CBT), third-wave CBT and interpersonal therapy (IPT) based interventions for preventing depression in children and adolescents. Cochrane Database Syst Rev 2016; 8: CD003380

[13] Jerstad S], Boutelle KN, Ness KK, Stice E. Prospective reciprocal relations between physical activity and depression in female adolescents. J Consult Clin Psychol 2010; 78: 268-272

[14] Kelsey MM, Zeitler PS. Insulin resistance of puberty. Curr Diab Rep 2016; 16: 1-6

[15] Kimm SY, Glynn NW, Kriska AM, Barton BA, Kronsberg SS, Daniels SR, Crawford PB, Sabry ZI, Liu K. Decline in physical activity in black girls and white girls during adolescence. N Engl J Med 2002; 347: 709-715

[16] Kovacs M. The Children's Depression Inventory (CDI). Psychopharmacol Bull 21: 995-998

[17] Kriska A, Delahanty L, Edelstein S, Amodei N, Chadwick J, Copeland K, Galvin B, El ghormli L, Haymond M, Kelsey M, Lassiter C, Mayer-Davis E, Milaszewski K, Syme A. Sedentary behavior and physical activity in youth with recent onset of type 2 diabetes. Pediatrics 2013; 131: e850-e856

[18] Lewinsohn PM, Clarke GN, Seeley JR, Rohde P. Major depression in community adolescents: age at onset, episode duration, and time to recurrence. J Am Acad Child Adolesc Psychiatry 1994; 33: 809-818 
[19] Luppino FS, de Wit LM, Bouvy PF, Stijnen T, Cuijpers P, Penninx BW, Zitman FG. Overweight, obesity, and depression: A systematic review and meta-analysis of longitudinal studies. Arch Gen Psychiatry 2010; 67: 220-229

[20] Marshall WA, Tanner JM. Variations in pattern of pubertal changes in girls. Arch Dis Child 1969; 44: 291-303

[21] Marshall WA, Tanner JM. Variations in the pattern of pubertal changes in boys. Arch Dis Child 1970; 45: 13-23

[22] Matthews DR, Hosker JP, Rudenski AS, Naylor BA, Treacher DF, Turner RC. Homeostasis model assessment: Insulin resistance and beta-cell function from fasting plasma glucose and insulin concentrations in man. Diabetologia 1985; 28: 412-419

[23] Nadeau KJ, Anderson B], Berg EG, Chiang JL, Chou H, Copeland KC, Hannon TS, Huang TT, Lynch JL, Powell J, Sellers E, Tamborlane WV, Zeitler P. Youth-onset type 2 diabetes consensus report: Current status, challenges, and priorities. Diabetes Care 2016; 39: 1635-1642

[24] Nader PR, Bradley RH, Houts RM, McRitchie SL, O'Brien M. Moderateto-vigorous physical activity from ages 9-15 years. JAMA 2008; 300: 295-305

[25] Norman AC, Drinkard B, McDuffie JR, Ghorbani S, Yanoff LB, Yanovski JA. Influence of excess adiposity on exercise fitness and performance in overweight children and adolescents. Pediatrics 2005; 115: e690-e696

[26] Radloff LS. The CES-D scale: A self report depression scale for research in the general population. 1977; Appl Psychol Meas 385-401

[27] Roberts MD, Drinkard B, Ranzenhofer LM, Salaita CG, Sebring NG, Brady SM, Pinchbeck C, Hoehl J, Yanoff LB, Savastano DM, Han JC, Yanovski JA. Prediction of maximal oxygen uptake by bioelectrical impedance analysis in overweight adolescents. J Sports Med Phys Fitness 2009; 49: 240-245
[28] Roshanaei-Moghaddam B, Katon W], Russo ]. The longitudinal effects of depression on physical activity. Gen Hosp Psychiatry 2009; 31: 306-315

[29] Shomaker LB, Kelly NR, Pickworth CK, Cassidy OL, Radin RM, Shank LM, Vannucci A, Thompson KA, Armaiz-Flores SA, Brady SM, Demidowich AP, Galescu OA, Courville AB, Olsen C, Chen KY, Stice E, Tanofsky-Kraff M, Yanovski JA. A randomized controlled trial to prevent depression and ameliorate insulin resistance in adolescent girls at risk for type 2 diabetes. Ann Behav Med 2016; 50: 762-774

[30] Shomaker LB, Kelly NR, Radin RM, Cassidy OL, Shank LM, Brady SM, Demidowich AP, Olsen CH, Chen KY, Stice E, Tanofsky-Kraff M, Yanovski JA. Prevention of insulin resistance in adolescents at risk for type 2 diabetes with depressive symptoms: 1-year follow-up of a randomized trial. Depress Anxiety 2017; 34: 866-876

[31] Shomaker LB, Tanofsky-Kraff M, Zocca JM, Field SE, Drinkard B, Yanovski JA. Depressive symptoms and cardiorespiratory fitness in obese adolescents. J Adolesc Health 2012; 50: 87-92

[32] Speilberger CD, Gorscuh RL, Lushene REl. Manual for the State-Trait Anxiety Inventory. California: Consulting Psychologists Press; 1970

[33] Titchener K, Wong QJ. A weighty issue: Explaining the association between body mass index and appearance-based social anxiety. Eat Behav 2015; 16: 13-16

[34] Zanoveli JM, Morais H, Dias IC, Schreiber AK, Souza CP, Cunha JM. Depression associated with diabetes: from pathophysiology to treatment. Curr Diabetes Rev 2016; 12: 165-178

[35] U.S. Department of Health and Human Services. Physical Activity Guidelines for Americans; $2^{\text {nd }}$ edition Available from: https://health. gov/paguidelines/second-edition/ 